\title{
Antisettici e controllo della carica batterica nelle ulcere venose Antiseptics and control of bacterial load in venous ulcers
}

\author{
Giuseppe Nebbioso, ${ }^{1}$ Ciro Falasconi, ${ }^{1}$ Viviana Nebbioso, ${ }^{2}$ Francesco Petrella ${ }^{3}$ \\ ${ }^{1}$ ASL NA1 Centro, Ambulatorio di Riparazione Tissutale; ${ }^{2}$ Facoltà di Chimica e Tecnologie Farmaceutiche, Università degli Studi di \\ Napoli “Federico II"; ${ }^{3}$ ASL NA3 Sud, Ambulatorio di Riparazione Tissutale, Napoli, Italia
}

\section{RIASSUNTO}

L'insorgenza di infezione su una lesione cutanea cronica determina un arresto del processo di riparazione tessutale e impone l'instaurazione di una terapia antibiotica sistemica che, in una fase iniziale, sarà empirica e, dunque, non scevra di insuccessi. L'utilizzo di antimicrobici con ridotto potere citotossoco/istiolesivo può essere considerata, in molti casi, una valida alternativa per il controllo della carica batterica e dell'infezione locale. L'associazione di antimicrobici a base di poliesanide biguanide, betaina e cadexomero iodico, in molti casi, permette di controllare/ridurre la carica batterica e l'infezione locale fungendo da starter per la ripresa del processo di riparazione tessutale.

\section{ABSTRACT}

The onset of infection on a skin ulcer (chronic wound) leads to a halt in the tissue repair process and requires a systemic antibiotic therapy which, at an early stage, will be empirical and, therefore, not free from setbacks. The use of antimicrobials with reduced cytotoxic/histiolesive power can be considered, in many cases, a valid alternative for bacterial burden and local infection control. The combination of antimicrobials based on polystyrene biguanide, betaine and iodine cadexomer, in many cases, allows to control/reduce the bacterial burden and local infection by acting as a starter for the resumption of the tissue repair process.

\section{INTRODUZIONE}

La guarigione delle lesioni croniche cutanee (LCC) avviene attraverso le fasi di emostasi, infiammazione, proliferazione e rimodellamento e può essere influenzato in maniera negativa da un aumento della carica batterica presente sulla lesione.

Corrispondenza: Giuseppe Nebbioso, ASL NA1 Centro, Ambulatorio di Riparazione Tissutale, Via Domenico Fontana 118, 20128 Napoli, Italia.

Tel.: +39.333.2440871

E-mail: giuseppe.nebbioso@gmail.com

Parole chiave: Antisettici; Wound Care; Ulcere venose.

Contributi: gli autori hanno contribuito equamente.

Conflitto d'interesse: gli autori dichiarano l'assenza di conflitti d'interesse.

Fondi: nesssuno.

Ricevuto per la pubblicazione: 19 febbraio 2018 .

Accettato per la pubblicazione: 8 maggio 2018.

This work is licensed under a Creative Commons Attribution NonCommercial 4.0 License (CC BY-NC 4.0).

CCopyright G. Nebbioso et al., 2018

Licensee PAGEPress, Italy

Italian Journal of Wound Care 2018; 2(2):40-44

doi:10.4081/ijwc.2018.21
La presenza di micro-organismi in concentrazioni significative, determina un prolungamento della fase infiammatoria con aumento persistente e duraturo delle metalloproteasi, del Tumor necrosis factor (TNF) e delle cellule della serie bianca con ulteriore danno tissutale.

Un'attenta valutazione della lesione, della cute perilesionale e della sintomatologia riferita dal paziente può dare utili informazioni su un eventuale aumento della carica batterica che, se non controllato in tempi rapidi, può evolvere verso un'infezione conclamata.

La comparsa di segni di infezione conclamata rende necessaria una terapia antibiotica che in fase iniziale, in attesa dei risultati dell'esame colturale, sarà di tipo empirico. Tale procedura non è però scevra da rischi, e può essere inefficace e generare fenomeni di antibiotico resistenza. ${ }^{1}$

Obiettivo dello studio è stato quello di valutare se il controllo della carica microbica potesse sbloccare il processo di guarigione di LCC che presentavano segni di flogosi locale e arresto del processo di cicatrizzazione nonostante il trattamento secondo i principi della wound bed preparation (WBP).

\section{MATERIALI E METODI}

Sono stati inclusi nello studio 12 pazienti con LCC, ad eziologia venosa che presentavano un ritardo di guarigione nonostante un corretto approccio terapeutico locale - WBP - con segni locali di probabile aumento della carica batterica.

Criteri di inclusione nello studio sono stati: i) Insor- 
genza, aumento o variazione del dolore; ii) Edema perilesionale; iii) Eritema perilesionale; iv) Tessuto di granulazione sanguinante o friabile; v) Aumento dell'essudato; vi) Pocketing; vii) Bridging.

Per pocketing si intende la presenza di aree lisce sul fondo della lesione senza granulazione ma circondate da tessuto di granulazione; per bridging ${ }^{2}$ si intende la presenza di filamenti o chiazze di tessuto formanti ponti che attraversano la lesione. $^{3}$

Criteri di esclusione sono stati: i) ulcere non venose; ii) malnutrizione; iii) uso di farmaci cortisonici e/o immunosoppressori; iv) terapia anticoagulante; v) coesistenza di epatopatia o nefropatia.

Le lesioni sono state medicate ogni giorno secondo il seguente schema terapeutico: i) Detersione del fondo della lesione con soluzione di poliesanide biguanide (PHMB) e betaina; ii) Applicazione di garza imbevuta di PHMB e betaina lasciata in situ per 10 minuti; iii) Cadexomero iodico in granuli apposto sul fondo della lesione; iv) Protezione del margine perilesionale con pasta all'ossido di zinco al 10\%; v) Garza sterile; vi) Bendaggio compressivo multistrato con bende a corta estensibilità; vii) Per la valutazione del dolore è stata utilizzata la scala di valutazione numerica (visual analogue scale, VAS), scala unidimensionale, rappresentata da una linea numerata e calibrata lunga $10 \mathrm{~cm}$ con ampiezza indicata da una serie di riquadri adiacenti.

\section{RISULTATI}

Dei 12 pazienti arruolati nello studio in 9 di essi si è avuto un miglioramento progressivo della lesione con una ripresa del processo di riparazione, con la scomparsa dei segni locali di flogosi e l'attenuazione/scomparsa della sintomatologia algica (Figure 1-4).

In particolare, nei valori della VAS dei 4 pazienti con sintomatologia dolorosa si osservavano i cambiamenti riportati nella Tabella 1 .

In 3 dei pazienti esaminati, a causa di un peggioramento della lesione, è stato necessaria la somministrazione di antibiotici, inizialmente con uno schema terapeutico di tipo empirico e successivamente sulla base dell'antibiogramma dopo esame colturale.

Tabella 1. Variazioni del punteggio visual analogue scale (VAS) nei 4 pazienti con sintomatologia dolorosa.

\begin{tabular}{lcc} 
Pariente & VAS iniziale & VAS finale \\
1 & 5 & 1 \\
\hline 2 & 4 & 0 \\
\hline 3 & 6 & 1 \\
\hline 4 & 5 & 0
\end{tabular}

\section{DISCUSSIONE}

Il processo di riparazione tissutale è un fenomeno alquanto complesso, articolato in diverse fasi: emostasi/infiammazione, proliferazione e rimodellamento. Durante queste fasi intervengono diversi elementi cellulari (piastrine, leucociti, fibroblasti) e molecole (fattori di crescita, interleukine, proteoglicani, glicomesoglicani) capaci di avviare e completare la guarigione della lesione. È possibile che in molti casi ci sia un rallentamento o un blocco del processo di riparazione, che trasforma la lesione da acuta in cronica.

I fattori responsabili di questa trasformazione possono essere distinti in: i) Locali: aumento della carica batterica (colonizzazione e infezione), presenza di slough e/o tessuto non vitale, iperessudazione, sede della lesione; ii) $\mathrm{Si}$ -

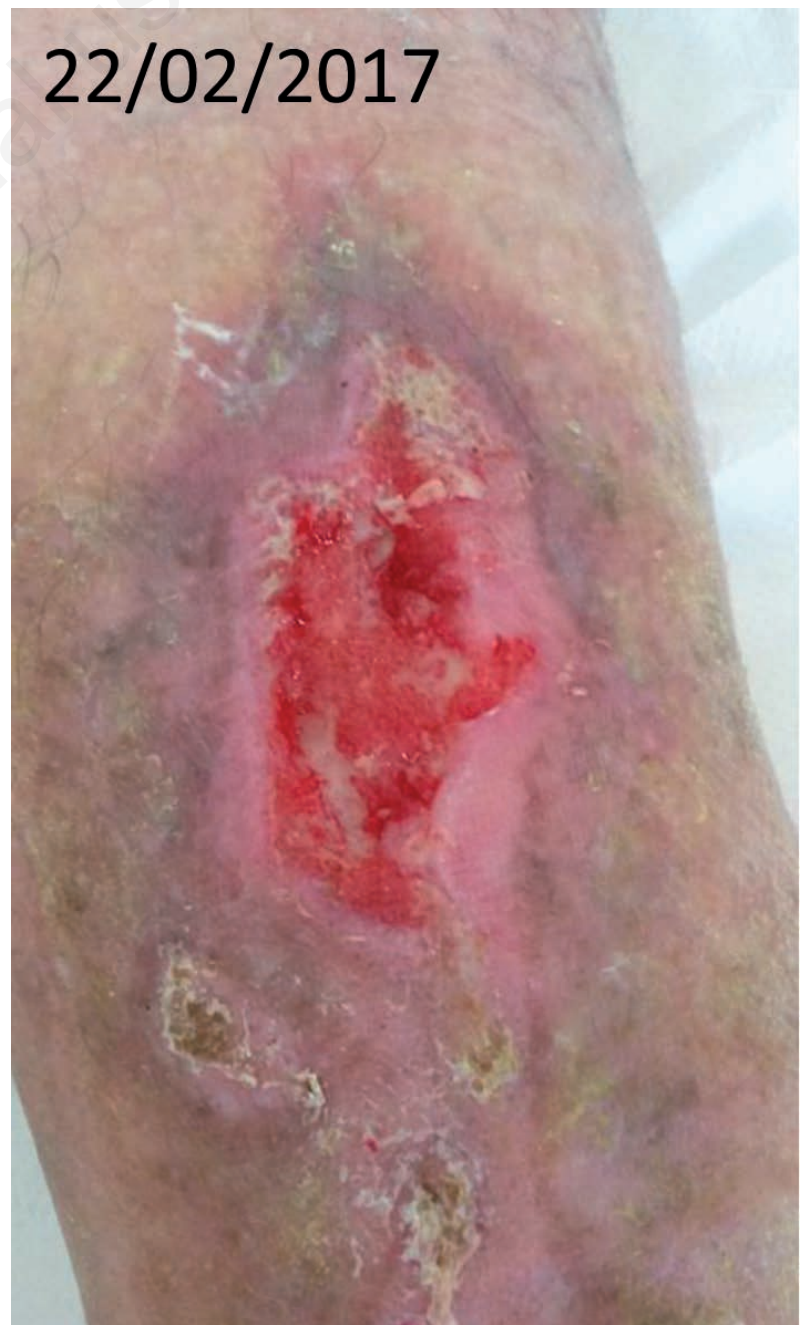

Figura 1. Paziente 1. 
stemici: malnutrizione, malattie metaboliche quali il diabete, immunodeficienze, uso di farmaci immunosoppressivi, vascolopatie; iii) L'infezione rappresenta una delle cause che più frequentemente blocca il processo di riparazione tessutale, ostacolando la guarigione della lesione ulcerativa e favorendone la cronicizzazione. La continua presenza di microorganismi virulenti porta a una risposta infiammatoria massiccia e persistente con aumento di citochine, dell'attività proteasica, del TNF, associata a ridotta attività dei fattori di crescita.

La formazione del biofilm sul fondo della lesione contribuisce al mantenimento dello stato flogistico, ostacolando l'azione degli antimicrobici sui microoganismi.

La presenza di microorganismi non è sempre sinonimo di infezione ed infatti in letteratura si fa distinzione tra contaminazione, colonizzazione critica ed infezione. Tutte

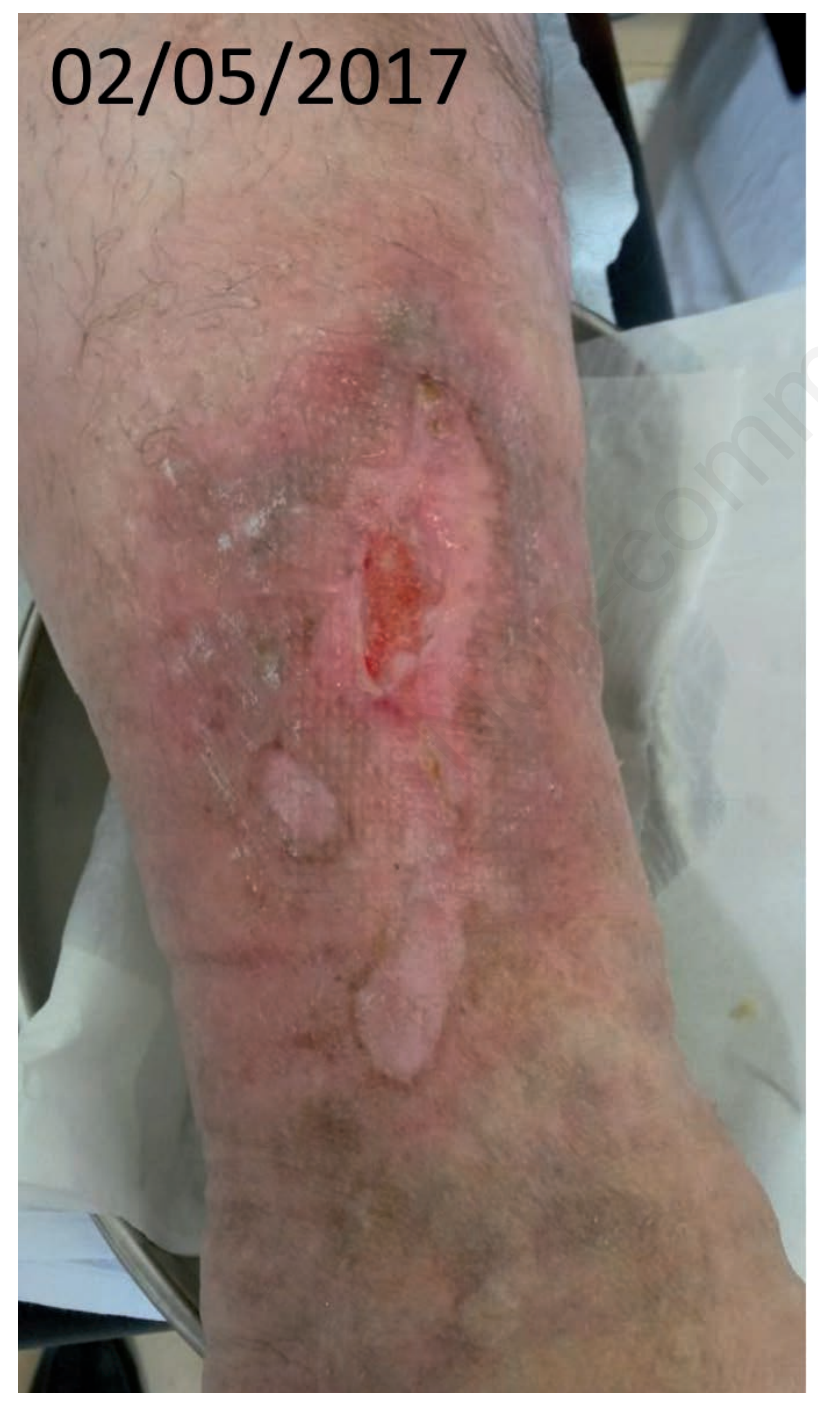

Figura 2. Paziente 2. le lesioni sono contaminate ma, in questi casi, la presenza di microorganismi non è caratterizzata da intensa crescita o replicazione con assenza di effetti sulla guarigione.

Al contrario, la presenza di microorganismi che si moltiplicano determina uno stato definito di colonizzazione critica che può ritardare la riparazione della ferita con segni perilesionali di flogosi. La replicazione massiva determina un chiaro stato di infezione con segni locali a rapida estensione e sintomatologia generale.

La gravità dell'azione lesiva dipende dalla virulenza e dalla specie batterica, dal numero di colonie presenti, dalle tossine eventualmente prodotte e dallo stato immunitario del paziente. È stato dimostrato che si verificano peggioramenti delle ulcere quando i batteri sono presenti in misura di $10^{5}-10^{6} \mathrm{CFU} / \mathrm{gr}$, indipendentemente dal tipo di batteri presenti. ${ }^{4}$

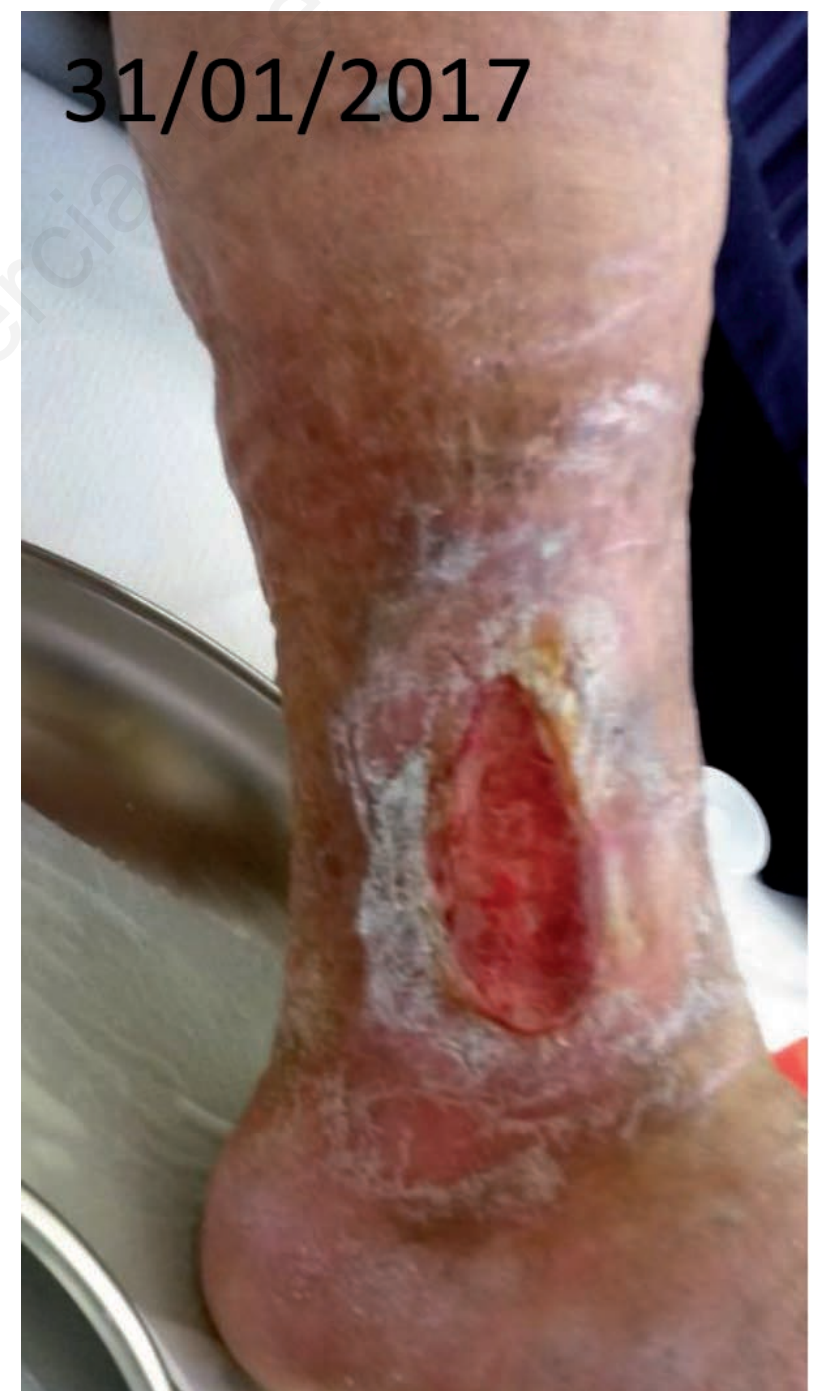

Figura 3. Paziente 3. 
Nella valutazione dello stato microbico, valutando i segni clinici, possiamo distinguere quattro stadi: i) Stadio1: lieve aumento dell'essudazione e dolore lieve; ii) Stadio 2: ulteriore aumento dell'essudazione, del dolore e comparsa di odore sgradevole (rallentamento o arresto guarigione); iii) Stadio 3: segni manifesti di infezione localizzata (secrezione purulenta, dolore, edema marcato); iv) Stadio 4: segni locali e sistemici (piressia, aumento dei leucociti, compromissione generale. ${ }^{5}$

L'identificazione precoce di un possibile e iniziale stato settico della lesione, stadio uno e due, è di importanza fondamentale al fine di evitare il blocco del processo di riparazione tessutale.

Per le ferite acute, in assenza di dati microbiologici, sono stati elaborati dei sistemi di punteggio e criteri diagnostici come l'ASEPSIS e le definizioni dei centers for

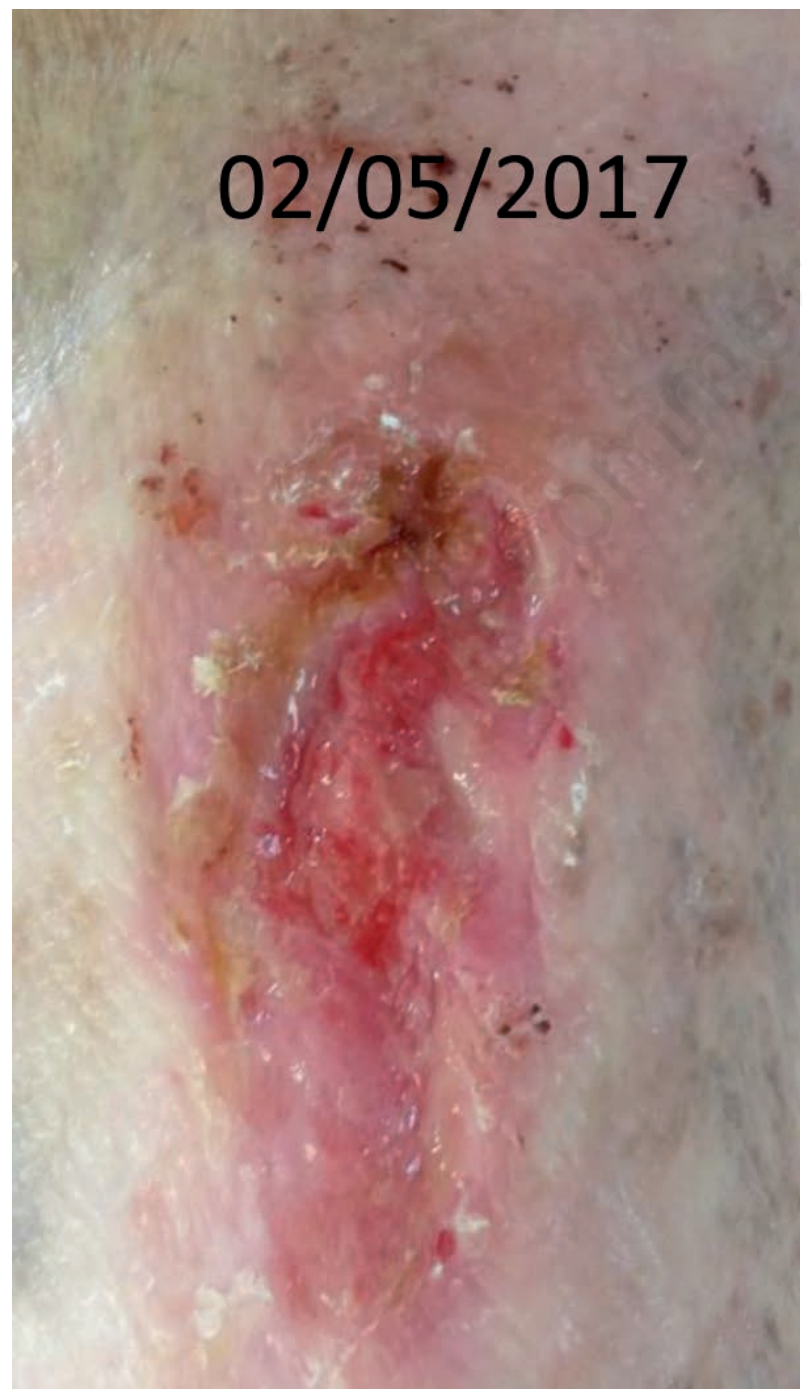

Figura 4. Paziente 4.
Disease Control and Prevention ma per le ferite croniche non ci sono criteri validati e ci si basa sui i caratteri locali della lesione e su criteri clinici. ${ }^{6}$

L'aumento della carica batterica determina la comparsa o il persistere di uno stato di infiammazione cronica che arresta la guarigione, pertanto l'utilizzo di antisettici, tramite un controllo dei microrganismi, può contribuire a controllare l'infezione.

Gli antisettici sono sostanze che inducono la morte o l'inibizione della crescita dei microrganismi nei tessuti vitali. ${ }^{7}$ L'antisettico ideale dovrebbe avere i seguenti requisiti: potenza e selettività di azione, attività anche in presenza di essudati infiammatori, assenza di tossicità sulla cute, mancanza di potere allergico. Prodotti di nuova generazione combinano l'azione tensioattiva senza essere istiolesivi. Tali prodotti vengono impiegati irrigando la lesione o sotto forma di impacco, lasciando cioè il prodotto sul sito di lesione per il tempo necessario a completare la sua funzione. ${ }^{8}$

È noto che l'accorsa di neutrofili nel letto della lesione determina il rilascio di peptidi antimicrobici naturali (AMP) che legandosi alle membrane cellulari dei batteri ne determinano la lisi e la morte. ${ }^{9}$

Il PHMB è un composto sintetico costituito da polimeri aventi analogie strutturali con gli AMP e per tal motivo può inserirsi nella struttura della membrana cellulare batterica, penetrare nella cellula, legarsi al DNA e ad altri acidi nucleici e provocarne la precipitazione. ${ }^{10}$

La Betaina, tensioattivo alcaloide anfoterico, riduce la tensione superficiale del letto di lesione migliorando il processo di detersione. L'associazione della Betaina e del PHMB nella preparazione del letto di lesione permette da un lato di rimuovere con più semplicità i tessuti non vitali e i detriti di lesione dall'altro di rompere il biofilm e agire sui microorganismi presenti riducendo e controllando la carica batterica e i segni di flogosi con riduzione del dolore e miglioramento della qualità di vita del paziente. ${ }^{11}$

L'utilizzo nel protocollo terapeutico di Cadexomero Iodico sul letto di lesione è dovuto alla sua capacità di danneggiare la struttura proteica cellulare dei patogeni attraverso la reazione con i loro amminoacidi con conseguente rottura sia della membrana cellulare di numerosi batteri, inclusi 103 isolati MRSA (in vitro) sia del biofilm. ${ }^{12,13}$

L'utilizzo del cadexomero permette, inoltre, sia la gestione dell'essudato (che viene inglobato nei granuli) sia il continuo debridement della lesione dovuto alla sua gelificazione (meccanismo autolitico).

\section{CONCLUSIONI}

L'arresto del processo di riparazione tessutale in una lesione, trattata secondo i principi della WBP, può essere legato a un aumento della carica batterica. L'incremento 
critico delle colonie sul fondo di lesione determina la comparsa di segni iniziali e locali di flogosi che possono essere considerati come campanello d'allarme di un'infezione in evoluzione. L'uso di antimicrobici a base di PHMB, betaina e cadexomero iodico permette, in molti casi, di controllare e ridurre l'incremento dei germi fungendo da starter per la ripresa del processo di riparazione tessutale.

\section{BIBLIOGRAFIA}

1. Hashem NG, Hidayat L, Berkowitz L, Venugopalan V. Management of skin and soft-tissue infections at a community teaching hospital using a severity-of-illness tool. J Antimicrob Chemother 2016; 71:3268-75.

2. Principi di Best Practice. Linee Guida Internazionali. III Congresso WUWH, Toronto, Canada; 2008.

3. Gardner SE, Hillis SL, Frantz RA. Clinical signs of infection in diabetic foot ulcers with high microbial load. Biol Res Nurs 2009;11:119-28.

4. Browne AC1, Vearncombe M, Sibbald RG. High bacterial load in asymptomatic diabetic patients with neurotrophic ulcers retards wound healing after application of Dermagraft. Ostomy Wound Manag 2001;47:44-9.
5. Metcalf DG, Bowler PG, Hurlow J. A clinical algorithm for wound biofilm identification. J Wound Care 2014;23:137-2.

6. Rondas AA, Schols JM. Definition of infection in chronic wounds by Dutch nursing home physicians. Int Wound J 2009;6:267-74.

7. Block SS. Definition of terms. In: Block SS, ed. Disinfection, Sterilization, and Preservation. Philadelphia: Lippincott Williams \& Wilkins; 2001. pp 19-28.

8. Bellingeri A, Falciani F, Traspedini P, et al. Effect of a wound cleansing solution on wound bed preparation and inflammation in chronic Wounds: a single-blind RCT. J Wound Care 2016;25:3:160-8.

9. Hancock RE, Sahl HG. Antimicrobial and host-defense peptides as new antiinfective therapeutic strategies. Nat Biotechnol 2006;24:1551-7.

10. Allen JM, White GF, Morby PA. The response of Escherichia coli to exposure to the biocide polyhexamethylene biguanide. Microbiology 2006;152:989-1000.

11. Bjarnsholt T, Cooper R. Gestione del Biofilm. Documento di posizionamento WUWHS. Wounds Edition; 2016.

12. Drosou A, Falabella A, Kirsner RS. Antiseptics on wounds: An area of controversy. Wounds 2003;15:149-66.

13. Phillips PL. Effects of antimicrobial agents on an (in-vitro) biofilm model of skin wounds. Adv Wound Care 2010; 1:299-304. 